Z Gerontol Geriat 2016 · 49:271-273

DOI 10.1007/s00391-016-1073-6

Online publiziert: 1. Juni 2016

๑) Springer-Verlag Berlin Heidelberg 2016

CrossMark

\section{Gisinger ${ }^{1,2} \cdot$ J. Pripfl ${ }^{3}$}

' Donau-Universität Krems, Krems an der Donau, Österreich

${ }^{2}$ Haus der Barmherzigkeit Wien, Wien, Österreich

${ }^{3}$ Akademie für Altersforschung, Haus der Barmherzigkeit Wien, Wien, Österreich

\title{
Alter schützt vor Technik nicht?!
}

Warum schützen? Seit der industriellen Revolution haben zahlreiche technische Errungenschaften den Alltag gerade auch von älteren Menschen erleichtert, z. B. Eisenbahn, Telefon, Haushaltsgeräte, Unterhaltungselektronik. Geht es nicht vielmehr umgekehrt darum, ob Technik vor Alter bzw. damit in Verbindung stehenden funktionellen Einschränkungen $\mathrm{zu}$ schützen vermag? Diese Frage kann klar bejaht werden, wenn wir an technische und medizintechnische Errungenschaften denken, wie z. B. die Endoprothetik. Schwerer einzuschätzen ist die mögliche Rolle von Assistenz- oder Servicerobotern, die von einigen Autorinnen und Autoren bereits als eine mögliche Lösung für den prognostizierten Arbeitskräftemangel im Pflegebereicht diskutiert werden [1-3]. Wie weit ist die Technik tatsächlich fortgeschritten? Was ist Realität? Was Fiktion? Welche ethischen Aspekte sind zu berücksichtigen?

Das vorliegende Heft der Zeitschrift für Gerontologie und Geriatrie mit dem Schwerpunktthema „Roboter für ältere Menschen" versucht, anhand von 5 Beiträgen Einblick in den derzeitigen Stand der Technik und Forschung zu geben.

Eftring et al. erläutern den aufwendigen Designprozess, der von Beginn an wesentlich auf die Einbeziehung von Endbenutzerinnen und Endbenutzern angewiesen ist. Dazu gehören Bedarfsund Bedürfniserhebung mithilfe von Workshops, Fragebogenerhebungen sowie qualitative Interviews mit älteren Menschen und deren Angehörigen, die den Ausgangspunkt für die Spezifikation von Aufgaben darstellen, die der Assistenzroboter „Hobbit" erfüllen soll. Die einzelnen Entwicklungsschritte umfassten Tests mit „mock-ups“ im Maßstab 1:1 in Privatwohnungen von Seniorinnen und Senioren, auf deren Basis schließlich ein erster Prototyp entwickelt wurde, der nach ausgiebigen Labortests zu einem wesentlich verbesserten zweiten Prototyp führte, welcher dann tatsächlich in realen Privatwohnungen einem mehrwöchigen Langzeittest unterzogen werden konnte. Dieser Schritt von Tests im Labor zum Einsatz eines sozialen Serviceroboters in privaten Haushalten ist weltweit beinahe einzigartig. Pripfl et al. berichten im zweiten Beitrag, nach einem kurzen Überblick über Gründe, warum es derzeit noch keine sozialen Serviceroboter am Markt zu kaufen gibt, über die Evaluierung des Feldversuchs, in dem der „Hobbit Prototyp 2“ bei sieben österreichischen Benutzerinnen und Benutzern für jeweils drei Wochen in deren privaten Haushalten zum Einsatz kam.

Ebenfalls von einem Feldversuch berichten Gerling et al. im dritten Beitrag. Die Autorinnen und Autoren setzten einen mobilen Roboter als Assistent im Bereich der Physiotherapie von Menschen mit fortgeschrittener Demenz ein und diskutieren anhand dieses Fallbeispiels Herausforderungen und Möglichkeiten, die mit der Integration von Technologien im Pflegekontext einhergehen.

Einer zentralen Frage für die künftige Entwicklung von Robotern für ältere Menschen gehen Panek et al. im vierten Beitrag nach: Sollten Assistenzroboter eine „Persönlichkeit" haben? Die Autoren entwickelten ein „Roboterpersönlichkeitsmodell“" und versuchen auf Basis von Ergebnissen einer empirischen Studie eine Antwort aufdiese Frage zu geben.

Im fünften Beitrag zeigt Körtner auf, dass es nicht für alle ethischen Herausforderungen beim Einsatz von sozial-as- sistiven Robotern in der Pflege oder im Heimgebrauch bereits klare Antworten gibt. Fragen zu Privatsphäre und Datenschutz, Sicherheit und Verantwortlichkeit sowie zum Umgang mit vulnerablen Personen und zur Täuschung werden vor dem Hintergrund ethischer Grundsätze diskutiert.

Dieses Schwerpunktthema gibt Einblick in den derzeitigen Stand der Entwicklung und Forschung und beleuchtet die ethischen Rahmenbedingungen des Einsatzes von assistierenden Robotern für ältere Menschen. Obwohl derzeit noch keine marktreifen Produkte verfügbar sind, ist es sicherlich nur eine Frage der Zeit, bis soziale Serviceroboter mit wertvollen Assistenzfunktionen Einzug in private Haushalte und den institutionalisierten Pflegebereich halten werden. Die eigentliche pflegerische Tätigkeit (z. B. Wundversorgung) sowie die psychosoziale Versorgung älterer Menschen werden aus Sicht der Editoren aber auch langfristig auf Mensch-zu-MenschInteraktion angewiesen sein.

Wir danken den beteiligten Autorinnen und Autoren für die inspirierenden Beiträge und wünschen den Leserinnen und Lesern viel Spaß beim Eintauchen in diese interdisziplinäre, zukunftsträchtige Thematik.

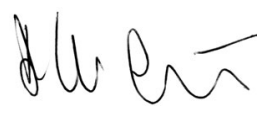

Univ.-Prof. Dr. C. Gisinger

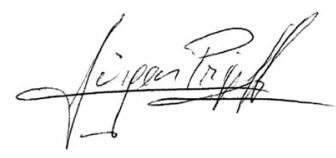

Mag. Dr. J. Pripfl 


\section{Editorial}

\section{Korrespondenzadresse}

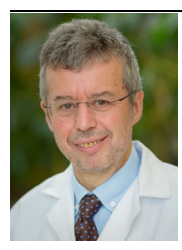

Univ.-Prof. Dr. C. Gisinger

Haus der Barmherzigkeit

Wien

Seeböckgasse 30A,

1160 Wien, Österreich

Christoph.Gisinger@

hausderbarmherzigkeit.at

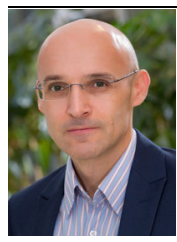

Mag. Dr. J. Pripfl

Akademie für Alters-

forschung, Haus der

Barmherzigkeit Wien

Seeböckgasse 30A,

1160 Wien, Österreich

Juergen.Pripfl@

altersforschung.ac.at

Interessenkonflikt. C. Gisinger und J. Pripfl geben

an, dass kein Interessenkonflikt besteht.

\section{Literatur}

1. Broadbent E, Stafford R, Macdonald B (2009) Acceptance of healthcare robots for the older population: review and future directions. Int J Soc Robot 1:319-330

2. Broekens J, Heerink M, Rosendal H (2009) Assistive social robots in elderly care: a review. Gerontechnology 8:94-103

3. Kachouie R, Sedighadeli S, Khosla R et al (2014) Socially Assistive robots in elderly care: A mixedmethod systematic literature review. Int J Hum Comput Interact 30:369-393 
Hier steht eine Anzeige.

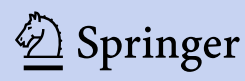

